\title{
Dynamical properties and melting of binary two-dimensional colloidal alloys
}

\author{
I. R. O. Ramos, ${ }^{1,2}$ W. P. Ferreira, ${ }^{1}$ F. F. Munarin, ${ }^{3}$ and F. M. Peeters ${ }^{1,4}$ \\ ${ }^{1}$ Departamento de Física, Universidade Federal do Ceará, Caixa Postal 6030, Campus do Pici, 60455-760 Fortaleza, Ceará, Brazil \\ ${ }^{2}$ Universidade Federal Rural do Semi-árido, Campus Pau dos Ferros, Pau dos Ferros, Rio Grande do Norte, Brazil \\ ${ }^{3}$ Centro de Tecnologia, Bloco 710, Universidade Federal do Ceará, Campus do Pici, 60455-760 Fortaleza, Ceará, Brazil \\ ${ }^{4}$ Department of Physics, University of Antwerp, Groenenborgerlaan 171, B-2020 Antwerpen, Belgium \\ (Received 11 March 2013; revised manuscript received 18 September 2014; published 22 December 2014)
}

\begin{abstract}
A two-dimensional (2D) binary colloidal system consisting of interacting dipoles is investigated using an analytical approach. Within the harmonic approximation we obtain the phonon spectrum of the system as a function of the composition, dipole-moment ratio, and mass ratio between the small and big particles. Through a systematic analysis of the phonon spectra we are able to determine the stability region of the different lattice structures of the colloidal alloys. The gaps in the phonon frequency spectrum, the optical frequencies in the longwavelength limit, and the sound velocity are discussed as well. Using the modified Lindemann criterion and within the harmonic approximation we estimate the melting temperature of the sublattice generated by the big particles.
\end{abstract}

DOI: 10.1103/PhysRevE.90.062311

PACS number(s): 82.70.Dd, 64.70.pv, 64.70.D-

\section{INTRODUCTION}

Colloids are useful model systems not only for important physical phenomena such as crystallization and melting [1,2] but also for solids, especially because of the orders-ofmagnitude slower temporal and larger spatial scales that allow the use of videomicroscopy techniques $[3,4]$ and, therefore, properties such as structure, phonons, and melting can be studied in real time [5]. There are several possibilities (e.g., at interfaces, in between glass plates, and patterned substrates) to stabilize the colloidal particles into a reduced dimensional system such as channels and planar substrates. Additionally, the interaction between the colloidal particles and thus the physical properties of the system can be externally controlled, e.g., by means of external magnetic [6] and/or electric fields [7].

Colloidal systems composed of two different types of dipolar particles confined in a monolayer structure have attracted the attention of many theoretical and experimental researchers. Stirner et al. [8] performed molecular dynamics simulations at finite temperatures of a binary colloidal monolayer of two different particle sizes at an oil-water interface whose interparticle interaction is governed by an effective dipole potential. The simulations showed that for certain ratios of small $(B)$ to large $(A)$ particles, e.g., 2:1 $\left(A B_{2}\right)$ and 6:1 $\left(A B_{6}\right)$, the system forms a two-dimensional (2D) crystal. In both cases the crystal is composed of a hexagonal lattice of large particles with a unit cell composed of one $A$ particle and two $B$ particles for $A B_{2}$ and one $A$ particle and six $B$ particles for $A B_{6}$. Studies of the zero-temperature $(T=0)$ phase diagram of a $2 \mathrm{D}$ binary system of dipoles using lattice-sum calculations [9] and genetic algorithms $[10,11]$ predicted the formation of several possible lattice structures as a function of the composition and the susceptibility ratio.

The structural behavior of binary mixtures of superparamagnetic colloidal particles at an air-water interface was investigated using integral equation theory together with computer simulations $[12,13]$ and experiments $[13,14]$. Those studies, however, found only a partial clustering of small particles [12,13] and a local crystalline order [14].

More recently, an interesting experimental and theoretical study [15] of a 2D binary colloidal system of large $(A)$ and small $(B)$ silica particles at an octane-water interface was presented as a function of the relative concentration of small particles $\xi=\rho_{B} /\left(\rho_{A}+\rho_{B}\right)$, where $\rho_{A}, \rho_{B}$ are the $2 \mathrm{D}$ densities of $A$ and $B$ particles, respectively. Due to the experimental setup, the particles were supposed to interact through a repulsive dipole-dipole potential and it was found that the system self-assemblies in a hexagonal alloy phase (HAP). Also, it was shown that while the HAP for $\xi=2 / 3\left(A B_{2}\right)$ and $\xi=6 / 7\left(A B_{6}\right)$ are thermodynamically stable, the HAP for $\xi=3 / 4\left(A B_{3}\right)$ and $\xi=5 / 6\left(A B_{5}\right)$ was unstable. A comparison between the radial distribution function of the small $B$ particles around the $A$ particles $g_{A B}(r)$ obtained from the analytical MEC with the one obtained from $T \neq 0$ Monte Carlo simulations, which were based on a finite-size computational unit cell, was further used in order to determine if the configuration was stable. The structure and melting behavior of the system was also studied theoretically as a function of the composition and the dipole-moment ratio, using a lattice-sum method and Monte Carlo simulations [16]. By investigating the radial distribution function for small particles $g_{B B}(r)$ as a function of temperature, it was found that the melting temperature of the $A B_{2}$ and $A B_{6}$ configurations was three orders of magnitude larger than that of the $A B_{5}$ structure [16].

In this work, we address the phonon spectrum and melting of a 2D binary colloidal system of dipoles which consists of particles with small and large dipole moments $\mu_{B}$ and $\mu_{A}$, respectively. Within the harmonic approximation we calculate the phonon spectrum of the system for different values of the dipole-moment ratio $s_{B}=\mu_{B} / \mu_{A}$, the relative concentration of small particles $\xi$, and mass ratio $m^{*}=m_{B} / m_{A}$. The motivation to do so is twofold: (1) it is possible to tune the number and width of the phonon gaps, and the shape of the phonon bands, by changing parameters such as the dipole-moment ratio, composition, and mass ratio [17] and (2) the study of the phonon spectra tells us additionally if the colloidal alloys are stable, i.e., have real phonon frequencies. Specifically, through a systematic analysis of the dispersion relation we determine the interval of values of $s_{B}$ for which the considered colloidal alloys are stable. Furthermore, the study of the dispersion relation allows us to obtain the sound velocity 


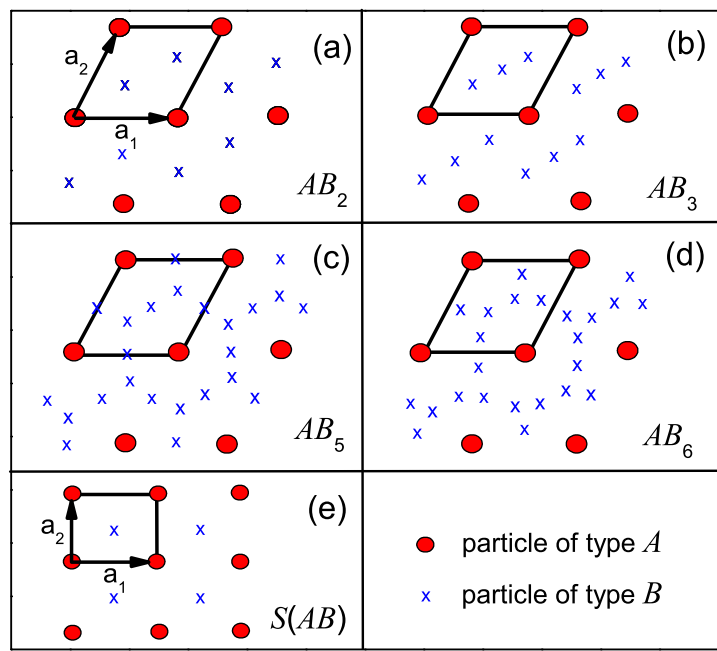

FIG. 1. (Color online) Structures of the colloidal alloys (a) $A B_{2}$, (b) $A B_{3}$, (c) $A B_{5}$, (d) $A B_{6}$, and (e) $S(A B)$. The unit cell of each phase is shown by the solid box and the primitive vectors are explicitly shown.

and the optical frequencies in the long-wavelength limit. We also present an estimation of the melting temperature of the sublattice generated by the big particles (type $A$ ) as a function of $s_{B}, \xi$, and $m^{*}$ using the modified Lindemann criterion. As a consequence, we found it is possible to specify the optimum value of $s_{B}$ for which the melting temperature of the system for a given composition is maximum.

In most part of this work, we will concentrate on the perfectly ordered 2D hexagonal colloidal alloys in order to model some of the configurations observed experimentally in Ref. [15], namely the configurations for $\xi=2 / 3\left(A B_{2}\right)$, $\xi=3 / 4\left(A B_{3}\right), \xi=5 / 6\left(A B_{5}\right)$, and $\xi=6 / 7\left(A B_{6}\right)$. Additionally, we also studied a 2D square alloy for $\xi=1 / 2$ $(S(A B))$. The lattice structures for these colloidal alloys are illustrated in Fig. 1. It is worth emphasizing that in our calculations we considered crystal structures, i.e., perfect periodic arrangements where defects and boundary effects are absent, in contrast with real experiments where, in general, defects, finite-size effects, or distortions of the crystal structure away from HAP might be present. Thus, although a small number of defects can be considered negligible from an experimental point of view (they may act as a stabilizing factor), they are determinant for the stability of the considered lattice from a theoretical point of view. For example, we find through analytical calculations (harmonic approach) that our perfect hexagonal alloy phase (HAP) for $\xi=2 / 3\left(A B_{2}\right)$ is not always stable even for the same set of parameters considered in the experiments [15], which indicates that some distortion with respect to the perfect HAP might be present in order to stabilize the experimentally observed configuration. Indeed, using Monte Carlo simulations (not shown), we find that a slightly distorted crystal structure with respect to the perfect HAP, which is similar to the one previously pointed out in Refs. [11,16], is observed as the ground state for the same value of the dipole-moment ratio $\left(s_{B} \approx 0.027\right)$ found analytically.

The paper is organized as follows. In Sec. II, we introduce the model, define the parameters used to characterize the system, and present the colloidal alloys considered in this work. In Sec. III, we present the calculation of the dispersion relation and discuss the numerical results. In Sec. IV, we study the melting behavior of the system. Our conclusions are given in Sec. V.

\section{MODEL}

We study a 2D binary colloidal system of dipole particles. The particles denoted by $A$ and $B$ have dipole moments $\mu_{A}$ and $\mu_{B}$, respectively, directed perpendicularly to the plane. The interaction potential is of the dipole-dipole form and can be written in two different ways:

$$
U_{k j}(r)=\frac{\mu_{k} \mu_{j}}{r^{3}}
$$

or

$$
\beta U_{k j}(r)=\Gamma \frac{s_{k} s_{j} a^{3}}{r^{3}},
$$

where $k, j=A, B$ and

$$
\Gamma=\frac{\mu_{A}^{2}}{k_{B} T a^{3}},
$$

is the dimensionless interaction strength, which relates the potential and the thermal energy, $r$ is the distance between two particles, $k_{B}$ the Boltzmann constant, $T$ the temperature of the system, $a$ the lattice parameter of the $A$ particles, $\beta=1 / k_{B} T$, and $s_{B}=\mu_{B} / \mu_{A}$ is the dipole-moment ratio.

For the experimental system studied in Ref. [15], $A$ and $B$ stand for the large and small synthetic amorphous silica particles with diameters $3.00 \pm 0.05 \mu \mathrm{m}$ and $1.00 \pm 0.05 \mu \mathrm{m}$, respectively, located at an octane-water interface. In this case, the dipoles are mainly due to the residual charges at the particle-oil interface, and the considered dipole-moment ratio was $s_{B}=0.037$. On the other hand, for the experimental setup considered in Refs. [13] and [14], $A$ and $B$ represent the large and small superparamagnetic colloidal particles, respectively, at a water-air interface. For instance, from Ref. [13], the big particles have diameter $4.7 \mu \mathrm{m}$, mass density $d_{A}=1.3 \mathrm{~g} / \mathrm{cm}^{3}$, and magnetic susceptibility $\chi_{A}=6.2 \times 10^{-11} \mathrm{Am}^{2} / \mathrm{T}$, while the small ones have diameter $2.8 \mu \mathrm{m}$, mass density $d_{B}=1.5 \mathrm{~g} / \mathrm{cm}^{3}$, and magnetic susceptibility $\chi_{B}=6.6 \times 10^{-12} \mathrm{Am}^{2} / \mathrm{T}$. An external magnetic field $\vec{B}$ applied perpendicularly to the water-air interface induces in each particle a magnetic moment $\vec{\mu}_{i}=\chi_{i} \vec{B}$, where $i=A, B$. Thus, the dipole-moment ratio is $s_{B} \approx 0.1$.

The colloidal alloy phases depend on the dipole-moment ratio as well as the relative concentration of small particles,

$$
\xi=\frac{\rho_{B}}{\rho_{A}+\rho_{B}},
$$

where $\rho_{A}$ and $\rho_{B}$ are the $2 \mathrm{D}$ densities of $A$ and $B$ particles, respectively.

In the present paper we will study the phonons and melting of (1) perfect 2D hexagonal colloidal alloys in order to model the configuration found in Ref. [15]: the hexagonal configurations for $\xi=2 / 3\left(A B_{2}\right), \xi=3 / 4\left(A B_{3}\right), \xi=5 / 6$ $\left(A B_{5}\right)$, and $\xi=6 / 7\left(A B_{6}\right)$; and (2) a $2 \mathrm{D}$ square alloy for $\xi=1 / 2(S(A B))$. 
The system at hand is $2 \mathrm{D}$ with unit cell having one $A$ particle and $n$ small $B$ particles. Therefore, the equilibrium positions of $A$ particles and of $n B$ particles are given by $\vec{R}_{A}=\vec{R}$, and $\vec{R}_{B_{i}}=\vec{R}+\vec{c}_{i}$, where $\vec{R}=l_{1} \vec{a}_{1}+l_{2} \vec{a}_{2}$ with $l_{1}, l_{2}$ integers, $\vec{a}_{1}, \vec{a}_{2}$ are the primitive vectors, $\vec{c}_{i}=\alpha_{i} \vec{a}_{1}+\beta_{i} \vec{a}_{2}$, where $\alpha_{i}, \beta_{i} \in(0,1)$ are determined by minimizing the energy for a given dipole-moment ratio, and $i=1, \ldots, n$. The primitive vectors of the hexagonal lattice are $\vec{a}_{1}=a(1,0)$ and $\vec{a}_{2}=a(1 / 2, \sqrt{3} / 2)$, while, for the square lattice, $\vec{a}_{1}=a(1,0)$ and $\vec{a}_{2}=a(0,1)$. Since the colloidal alloys considered here have only one $A$ particle per unit cell, the density of $A$ particles $\rho_{A}$ is given by $\rho_{A} a^{2}=2 / \sqrt{3}$ and $\rho_{A} a^{2}=1$ for hexagonal and square unit cells, respectively.

\section{PHONON SPECTRUM}

The phonon spectrum will be calculated within the harmonic approximation. In this approach, one considers that each particle executes small oscillations (compared to the average distance between the particles) around its equilibrium position and, therefore, one expands the potential energy up to the second order in the deviations from its equilibrium position. Due to the periodicity of the system, one introduces Bloch plane wavelike solutions and thus one obtains (for a given wave vector $\vec{q}$ along the high-symmetry directions of the first Brillouin zone) the dynamical matrix whose eigenvalues and eigenvectors are the square frequencies of vibration, $\omega^{2}(\vec{q}, j)$, and the direction of vibration, $\vec{e}(\vec{q}, j)$, respectively, with $j=1, \ldots, 2 n_{p}$, where $n_{p}$ is the total number of particles per unit cell.

The study of the dispersion relation gives us additionally the stability of the mentioned colloidal alloys, for a given dipole-moment ratio $s_{B}$. The considered crystal structure of the colloidal alloy is stable only if $\omega^{2}(\vec{q}, j) \geqslant 0$ for all $\vec{q}$ and $j$ [18-22]. For $\omega^{2}(\vec{q}, j)<0$ the frequencies are imaginary, i.e., the amplitude of particle oscillation becomes an exponentially increasing function of time [22]. It implies that the corresponding crystal structure of the colloidal alloy is unstable and will not exist.

All the colloidal alloys considered in this work have more than one particle per unit cell. As a consequence, there are several acoustical and optical modes which can be associated to in-phase and out-of-phase vibrations of particles in the unit cell, respectively. The acoustical branch is characterized by $\omega(\vec{q}) \rightarrow 0$ for $\vec{q} \rightarrow 0$, while for the optical branch $\omega(\vec{q}) \rightarrow$ const in the limit $\vec{q} \rightarrow 0$. Besides, the acoustical and optical branches have a longitudinal, $\vec{e} \| \vec{q}$, and a transverse mode, [22] $\vec{e} \perp \vec{q}$.

The dynamical matrix is given by $[19,22,23]$

$$
C_{\alpha \beta}\left(k k^{\prime} \mid \vec{q}\right)=\frac{1}{\sqrt{m_{k} m_{k^{\prime}}}} \sum_{l^{\prime}} \phi_{\alpha \beta}\left(l k, l^{\prime} k^{\prime}\right) e^{-i \vec{q} .\left(\vec{R}_{l k}-\vec{R}_{l^{\prime} k^{\prime}}\right)}
$$

where $\phi(r)$ is the interaction potential and

$$
\phi_{\alpha \beta}\left(l k, l^{\prime} k^{\prime}\right)=\partial_{\alpha} \partial_{\beta} \phi\left(\vec{R}_{l k}-\vec{R}_{l^{\prime} k^{\prime}}\right)
$$

are the force constants with $\alpha, \beta=x, y$. Furthermore, $\vec{R}_{l k}=$ $\vec{R}(l)+\vec{R}(k)$ is the equilibrium position vector of the $k$ th particle in the $l$ th unit cell of the crystal, $m_{k}$ is its mass, and
$\vec{R}(l)=\vec{R}$. Besides, the force constants have the property

$$
\sum_{l k, l^{\prime} k^{\prime}} \phi_{\alpha \beta}\left(l k, l^{\prime} k^{\prime}\right)=0
$$

which will be useful in our further calculations. Thus, the equilibrium positions of $A$ particles and of $n B$ particles are given by $\vec{R}_{l A}=\vec{R}_{A}$ and $\vec{R}_{l B_{i}}=\vec{R}_{B_{i}}$. Furthermore, the order of the dynamical matrix is $t=2 n_{p} \times 2 n_{p}$, i.e., it depends on the considered 2D lattice. The dynamical matrix can be written as

$$
D=\left(\begin{array}{cccc}
D^{A A} & D^{A B_{1}} & \ldots & D^{A B_{n}} \\
D^{B_{1} A} & D^{B_{1} B_{1}} & \ldots & D^{B_{1} B_{n}} \\
\cdot & \cdot & \ldots & \cdot \\
\cdot & \cdot & \ldots & \cdot \\
\cdot & \cdot & \ldots & \cdot \\
D^{B_{n} A} & D^{B_{n} B_{1}} & \ldots & D^{B_{n} B_{n}}
\end{array}\right)
$$

where $D^{A A}, D^{A B_{1}}, \ldots, D^{B_{n} B_{n}}$ are $2 \times 2$ block matrices. From Eqs. (5) and (7), the elements of the block $D^{A A}$ are written by

$$
D_{\alpha \beta}^{A A}(\vec{q})=\frac{1}{m_{A}}\left[S_{\alpha \beta}^{A A}(0)+\sum_{i} S I 1_{\alpha \beta}^{A B_{i}}(0)-S_{\alpha \beta}^{A A}(\vec{q})\right],
$$

where

$$
\begin{gathered}
S_{\alpha \beta}^{A A}(\vec{q})=-\mu_{A}^{2} \lim _{r \rightarrow 0} \partial_{\alpha} \partial_{\beta} \psi_{0}(\vec{r}, \vec{q}), \\
\operatorname{SI}_{\alpha \beta}^{A B_{i}}(\vec{q})=-s_{B} \mu_{A}^{2} \lim _{r \rightarrow 0} \partial_{\alpha} \partial_{\beta} \psi_{I 1}^{i}(\vec{r}, \vec{q}),
\end{gathered}
$$

with

$$
\begin{aligned}
\psi_{0}(\vec{r}, \vec{q}) & =\sum_{\vec{R} \neq \overrightarrow{0}} \frac{e^{-i \vec{q} \cdot \vec{R}}}{|\vec{r}+\vec{R}|^{3}}, \\
\psi_{I 1}^{i}(\vec{r}, \vec{q}) & =\sum_{\vec{R}} \frac{e^{-i \vec{q} \cdot\left(\vec{R}+\vec{c}_{i}\right)}}{\left|\vec{r}+\vec{R}+\vec{c}_{i}\right|^{3}} .
\end{aligned}
$$

On the other hand, from Eq. (5) we found

$$
D_{\alpha \beta}^{A B_{i}}(\vec{q})=\frac{1}{\sqrt{m_{A} m_{B}}}\left[-S I 1_{\alpha \beta}^{A B_{i}}(\vec{q})\right] .
$$

In the same spirit of Refs. [18-20], we used the Ewald summation technique and transformed Eqs. (11a) and (11b) into expressions which converge rapidly. Thus, we obtain [20]

$$
\begin{aligned}
\psi_{0}(\vec{r}, \vec{q})= & \pi \rho_{A} \sum_{\vec{G}} e^{i(\vec{q}+\vec{G}) \cdot \vec{r}} \Upsilon\left(\frac{|\vec{q}+\vec{G}|}{2 \varepsilon}, 0\right)+\frac{2 \varepsilon e^{-\varepsilon^{2} r^{2}}}{\sqrt{\pi} r^{2}} \\
& -\frac{\operatorname{erf}(\varepsilon r)}{r^{3}}+\sum_{\vec{R} \neq \overrightarrow{0}} e^{-i \vec{q} \cdot \vec{R}} \Omega_{1}(|\vec{r}+\vec{R}|),
\end{aligned}
$$

with

$$
\begin{aligned}
& \Upsilon\left(\frac{|\vec{q}+\vec{G}|}{2 \varepsilon}, 0\right) \\
& \quad=\frac{4 \varepsilon}{\sqrt{\pi}} e^{-|\vec{q}+\vec{G}|^{2} / 4 \varepsilon^{2}}-2|\vec{q}+\vec{G}| \operatorname{erfc}\left(\frac{|\vec{q}+\vec{G}|}{2 \varepsilon}\right)
\end{aligned}
$$

and

$$
\Omega_{1}(x)=\frac{\operatorname{erfc}(\varepsilon x)}{x^{3}}+\frac{2 \varepsilon}{\sqrt{\pi}} \frac{e^{-\varepsilon^{2} x^{2}}}{x^{2}}
$$


where the parameter $\varepsilon>0$ is related to the density of large particles, i.e., $\varepsilon=\sqrt{\pi \rho_{A}}$. Besides, we have

$$
\begin{aligned}
\psi_{I 1}^{i}(\vec{r}, \vec{q})= & \pi \rho_{A} \sum_{\vec{G}} e^{i(\vec{q}+\vec{G}) \cdot \vec{r}} e^{i \vec{G} \cdot \vec{c}_{i}} \Upsilon\left(\frac{|\vec{q}+\vec{G}|}{2 \varepsilon}, 0\right) \\
& +\sum_{\vec{R}} e^{-i \vec{q} \cdot\left(\vec{R}+\vec{c}_{i}\right)} \Omega_{1}\left(\left|\vec{r}+\vec{R}+\vec{c}_{i}\right|\right)
\end{aligned}
$$

and, therefore, the block matrices $D^{A A}$ and $D^{A B_{i}}$ involve only rapidly convergent sums. On the other hand, the block $D^{B_{i} B_{j}}$, $i \neq j$, and $D^{B_{i} B_{i}}$ are written as

$$
\begin{aligned}
D_{\alpha \beta}^{B_{i} B_{j}}(\vec{q})= & \frac{1}{m_{B}}\left[-S I I 1_{\alpha \beta}^{B_{i} B_{j}}(\vec{q})\right], \\
D_{\alpha \beta}^{B_{i} B_{i}}(\vec{q})= & \frac{1}{m_{B}}\left[s_{B}^{2} S_{\alpha \beta}^{A A}(0)+\sum_{j \neq i} S I I 1_{\alpha \beta}^{B_{i} B_{j}}(0)\right. \\
& \left.+S I 1_{\alpha \beta}^{A B_{i}}(0)-s_{B}^{2} S_{\alpha \beta}^{A A}(\vec{q})\right],
\end{aligned}
$$

with

$$
\begin{aligned}
S I I 1_{\alpha \beta}^{B_{i} B_{j}}(\vec{q}) & =-s_{B}^{2} \mu_{A}^{2} \lim _{r \rightarrow 0} \partial_{\alpha} \partial_{\beta} \psi_{I I 1}^{i j}(\vec{r}, \vec{q}), \\
\psi_{I I 1}^{i j}(\vec{r}, \vec{q}) & =\sum_{\vec{R}} \frac{e^{-i \vec{q} \cdot\left(\vec{R}+\vec{c}_{i j}\right)}}{\left|\vec{r}+\vec{R}+\vec{c}_{i j}\right|^{3}} \\
\vec{c}_{i j} & =\vec{c}_{i}-\vec{c}_{j} .
\end{aligned}
$$

Again, the expression for $\psi_{I I 1}^{i j}(\vec{r}, \vec{q})$ using the Ewald method is given by

$$
\begin{aligned}
\psi_{I I 1}^{i j}(\vec{r}, \vec{q})= & \pi \rho_{A} \sum_{\vec{G}} e^{i(\vec{q}+\vec{G}) \cdot \vec{r}} e^{i \vec{G} \cdot \vec{c}_{i j}} \Upsilon\left(\frac{|\vec{q}+\vec{G}|}{2 \varepsilon}, 0\right) \\
& +\sum_{\vec{R}} e^{-i \vec{q} \cdot\left(\vec{R}+\vec{c}_{i j}\right)} \Omega_{1}\left(\left|\vec{r}+\vec{R}+\vec{c}_{i j}\right|\right) .
\end{aligned}
$$

Since the dynamical matrix is Hermitian, we have $D^{B_{i} A}=$ $\left[D^{A B_{i}}\right]^{\dagger}$ and $D^{B_{j} B_{i}}=\left[D^{B_{i} B_{j}}\right]^{\dagger}$. Because the dynamical matrix involves the mass of the particles, we introduced the parameter $m^{*}=m_{B} / m_{A}$. For Brownian systems one can consider $m^{*}=1$, i.e., the particles have the same mass, since the inertial asymmetry between the colloids becomes irrelevant in the overdampped regime $[6,17,24]$. On the other hand, assuming that the dipole moment of each particle is $\mu_{i}=\lambda D_{i}^{3}$, where $\lambda$ is a constant of proportionality and $D_{i}$ is the radius of the particle [10], and that the particles have the same mass density, we obtain $m^{*}=s_{B}=\mu_{B} / \mu_{A}$. The case with different masses is available experimentally in systems of colloids between glass plates with no solvent [25]. In what follows, we will restrict ourselves to the two cases $m^{*}=s_{B}$ and $m^{*}=1$.

Figures 2 and 3 show the square of the phonon frequencies in units of $\omega_{0}^{2}=\mu_{A}^{2} \rho_{A}^{5 / 2} / m_{A}$ of the structure $A B_{2}$ [Fig. 1(a)] for $s_{B}=0.015$ and $s_{B}=0.037$, considering $m^{*}=s_{B}$ and $m^{*}=1$, respectively. The square frequencies are shown along the high-symmetry directions in reciprocal space, where the high-symmetry points are shown as insets. For $s_{B}=0.015$,

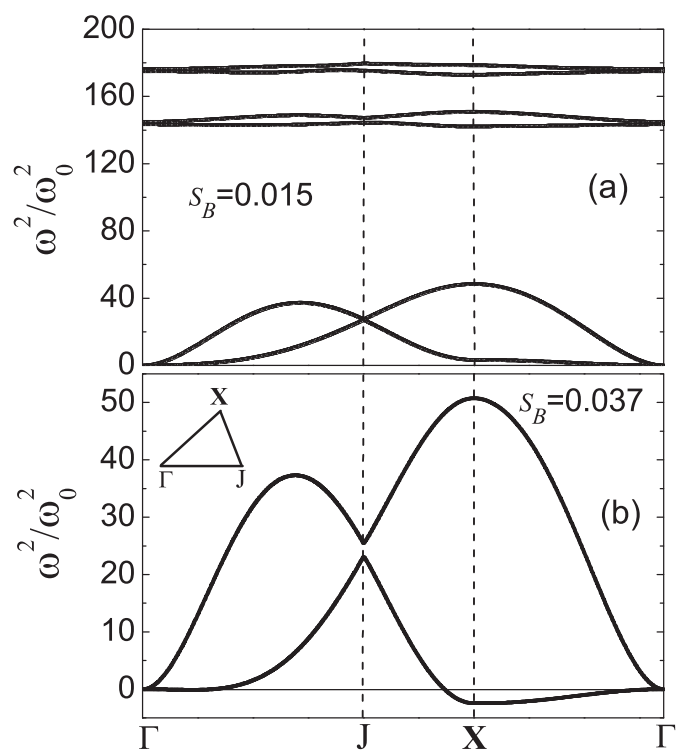

FIG. 2. Square of the phonon frequencies of the crystal phase $A B_{2}$ for $m^{*}=s_{B}$ in units of $\omega_{0}^{2}=\mu_{A}^{2} \rho_{A}^{5 / 2} / m_{A}$ (a) for $s_{B}=0.015$ and (b) $s_{B}=0.037$, along the high-symmetry directions in reciprocal space. The high-symmetry points $\Gamma, J$, and $X$ are shown in the inset of (b). Only the lowest energy modes are shown in (b) in order to enlarge the region around zero frequency.

we found $\omega^{2}(\vec{q}, j) \geqslant 0$ for all the eigenvalues, indicating a stable long-range $A B_{2}$ ordered structure. On the other hand, for the dipole-moment ratio $s_{B}=0.037$ considered in the experiments performed by Law et al. [15], we found $\omega^{2}(\vec{q}, j)<$ 0 for some eigenvalues, indicating that the perfect hexagonal $A B_{2}$ structure is not stable for $s_{B}=0.037$. Actually, we found that on the basis of the requirement of real phonon frequencies, the range of stability for the phase $A B_{2}$ is $0 \leqslant s_{B} \leqslant 0.0269$. We stress that in our calculations the considered perfect

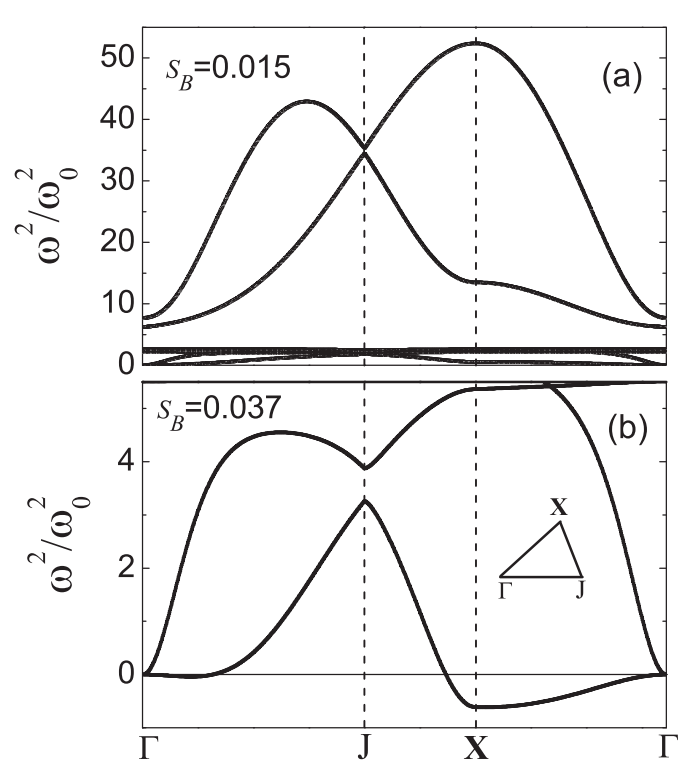

FIG. 3. Square of the phonon frequencies of the phase $A B_{2}$ for $m^{*}=1$ (a) for $s_{B}=0.015$ and (b) $s_{B}=0.037$. Only the lowest energy modes are shown in (b). 


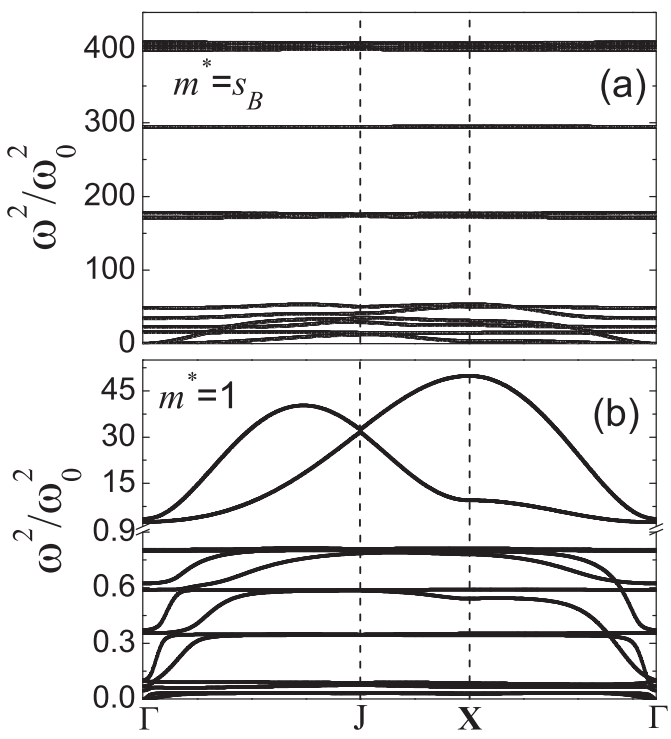

FIG. 4. Square of the phonon frequencies of the phase $A B_{6}$ for $s_{B}=0.002$ in units of $\omega_{0}^{2}=\mu_{A}^{2} \rho_{A}^{5 / 2} / m_{A}$ for (a) $m^{*}=s_{B}$ and (b) $m^{*}=1$.

periodic structures are free of defects and boundary effects, in contrast with the situation in real experiment where, in general, defects and imperfections might be present. Thus, although a small number of defects in the configuration $A B_{2}$ for $s_{B}=0.037$ can be considered negligible from an experimental point of view, they can be determinative for the stability from a theoretical point of view. Our results indicate that the stable phase observed experimentally in Ref. [15] must present some distortion from the perfect hexagonal alloy phase. As a consequence, we did not find long-range order
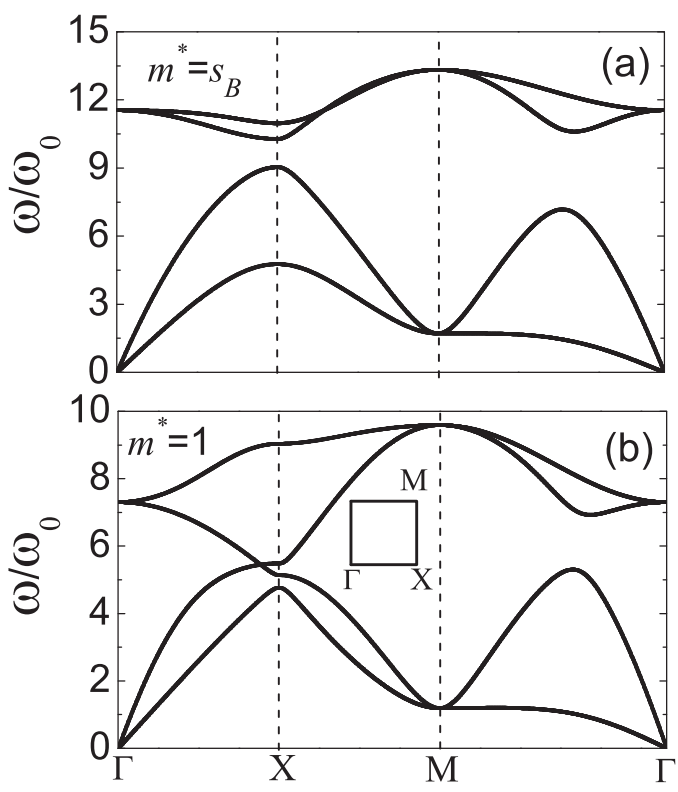

FIG. 5. Dispersion relation of the phase $S(A B)$ for $s_{B}=0.25$ along the high-symmetry directions in reciprocal space (a) for $m^{*}=$ $s_{B}$ and (b) for $m^{*}=1$. The high-symmetry points $\Gamma, X$, and $M$ are shown in the inset of (b).
TABLE I. Interval of stability of some colloidal alloys. The phases $A B_{3}$ and $A B_{5}$ are unstable and therefore are not listed.

\begin{tabular}{cccc}
\hline \hline Phases & $A B_{2}$ & $A B_{6}$ & $S(A B)$ \\
\hline Stable & $0 \leqslant s_{B} \leqslant 0.0269$ & $0 \leqslant s_{B} \leqslant 0.0043$ & $0.038 \leqslant s_{B} \leqslant 0.29$ \\
\hline \hline
\end{tabular}

for the perfect HAP $A B_{2}$ for $s_{B}=0.037$, but we found it for $s_{B}=0.0269$, which is a slightly lower value. Indeed, we performed Monte Carlo simulations for the specific phase $A B_{2}$ (not shown) and found that a slightly distorted crystal structure with respect to the perfect HAP which is also similar to the one previously pointed out in Refs. $[11,16]$ is observed as the ground state for the same value of the dipole-moment ratio $\left(s_{B} \approx 0.027\right)$ found analytically.

The presence of gaps in the phonon frequency spectrum is another important characteristic of the structure $A B_{2}$. No vibrations are possible for frequencies within the gap. The phonon gaps of the phase $A B_{2}$ for $m^{*}=s_{B}$, i.e., when the particles have different masses, are larger than the ones for $m^{*}=1$. Furthermore, for $m^{*}=s_{B}$, the phonon gaps occur between acoustical and optical modes as well as between some of the optical modes, while for $m^{*}=1$ the phonon gaps appear only between the optical modes. The thick line in Fig. 3 is due to two optical branches very close to each other that are not distinguishable on the scale used in the figure.

For the structures $A B_{3}$ and $A B_{5}$ shown in Figs. 1(b) and $1(\mathrm{c})$, respectively, we found that they are unstable for any dipole-moment ratio, since imaginary phonon frequencies are found. In other words, long-range order is not possible for the configurations $A B_{3}$ and $A B_{5}$ independently of the value of the dipole-moment ratio. Since in the harmonic approximation, the particles execute only small vibrations around their equilibrium positions, one cannot state that the structures $A B_{3}$ and $A B_{5}$ are stable at $T=0$. Our result clearly shows that calculations of the energy of a given lattice structure, even after minimization with respect to some parameters, at $T=0$, do not guarantee that the obtained MECs are stable.

In Fig. 4 we present the square of the phonon frequencies of the phase $A B_{6}$ for $s_{B}=0.002$. For the $A B_{6}$ configuration, we found that the interval of stability is $0 \leqslant s_{B} \leqslant 0.0043$.

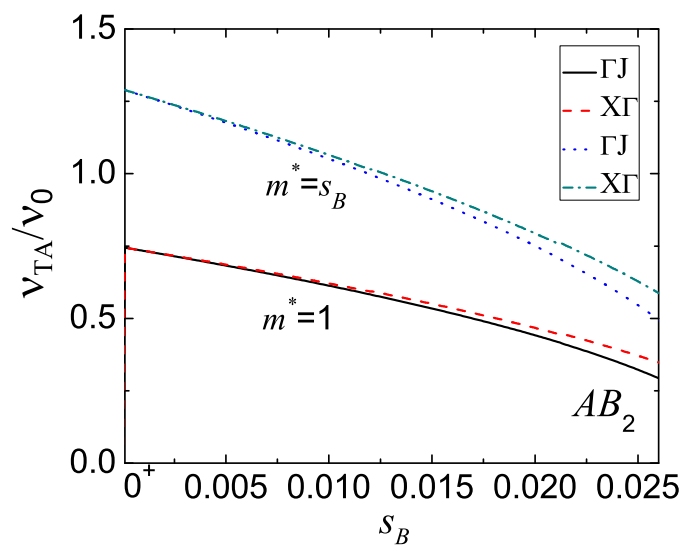

FIG. 6. (Color online) The sound velocity in units of $v_{0}=$ $\omega_{0} / \sqrt{\rho_{A}}$ of the transverse acoustical mode of the phase $A B_{2}$ as a function of $s_{B}$ for $m^{*}=1$ and $m^{*}=s_{B}$. 


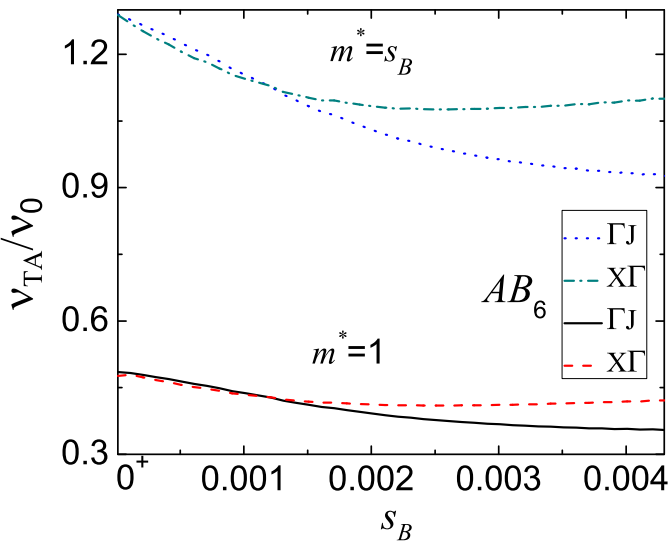

FIG. 7. (Color online) The sound velocity in units of $v_{0}=$ $\omega_{0} / \sqrt{\rho_{A}}$ of the transverse acoustical mode of the phase $A B_{6}$ as a function of $s_{B}$ for $m^{*}=1$ and $m^{*}=s_{B}$.

Therefore, if we considered not only the large A particles, there is no long-range $A B_{6}$ order for $s_{B}=0.037$ which is consistent with the experiments reported in Ref. [15]. However, the most important result for the $A B_{6}$ configuration is the considerable increase of the phonon gaps between the optical modes when the particles have different masses. As a consequence, there is a large number of frequencies for which the $A B_{6}$ structure cannot sustain vibrations. On the other hand, when the particles have the same mass, only a small phonon gap is found, between the optical modes, similarly to the phase $A B_{2}$.

Figure 5 shows the dispersion relation of the configuration $S(A B)$ for $s_{B}=0.25$. Again, on the basis of the requirement of real phonon frequencies, we found that the interval of stability of the alloy $S(A B)$ is $0.038 \leqslant s_{B} \leqslant 0.29$. Interestingly, in this case, we do not have stability for $s_{B}=0$, i.e., when only one particle is present in the unit cell. It is well known that a $2 \mathrm{D}$ system of particles interacting through a Coulomb potential when arranged in a square Bravais lattice is unstable [18]. Here, the same conclusion is reached when the particles interact through a repulsive dipole-dipole potential. Unlike the phases $A B_{2}$ and $A B_{6}$ that have phonon gaps for $m^{*}=s_{B}$ and $m^{*}=1$,

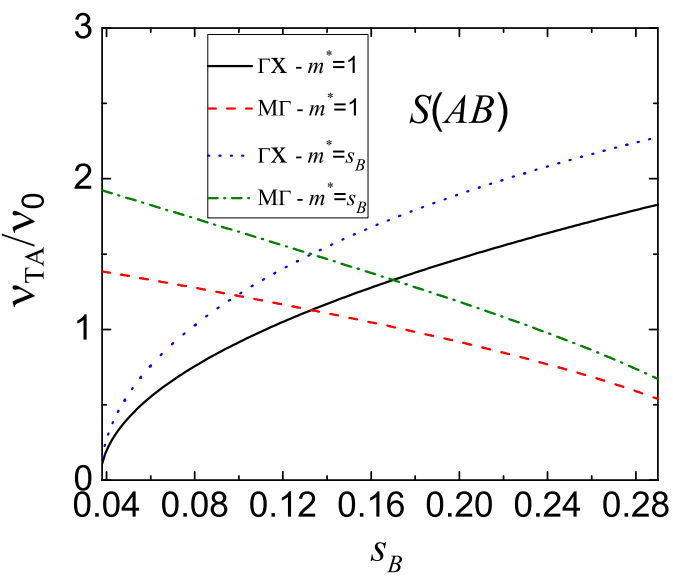

FIG. 8. (Color online) The sound velocity in units of $v_{0}=$ $\omega_{0} / \sqrt{\rho_{A}}$ of the transverse acoustical mode of the $S(A B)$ as a function of $s_{B}$ for $m^{*}=1$ and $m^{*}=s_{B}$.
TABLE II. Fitting parameters [Eq. (19)] for the sound velocity of the phase $A B_{2}$.

\begin{tabular}{lcccc}
\hline \hline$A B_{2}$ & $m^{*}=s_{B}$ & $m^{*}=s_{B}$ & $m^{*}=1$ & $m^{*}=1$ \\
\hline Direction & $\Gamma J$ & $X \Gamma$ & $\Gamma J$ & $X \Gamma$ \\
$v_{0}$ & 1.289 & 1.289 & 0.744 & 0.743 \\
$v_{1}$ & -17.38 & -18.32 & -9.166 & -9.745 \\
$v_{2}$ & -473.1 & -314.4 & -294.8 & -200.0 \\
\hline \hline
\end{tabular}

the configuration $S(A B)$ exhibits gaps in the phonon spectrum only for $m^{*}=s_{B}$. This is an example of how the properties of the system depend on the composition $\xi$. The interval of stability of some of the colloidal alloys are reported in Table I.

The sound velocity of the transverse acoustical (TA) mode, $v_{T A}=d \omega_{T A} /\left.d q\right|_{q \rightarrow 0}$, is shown for the stable configurations $A B_{2}, A B_{6}$, and $S(A B)$, in Figs. 6, 7, and 8, respectively, along the directions $(1,0)$ and $(1,1)$ (in what follows, the symbol $0^{+}$ means that we are not considering $s_{B}=0$ but only $s_{B}$ values very close to zero). For these phases, the sound velocity is large in the case the particles have different masses. For the structure $A B_{2}$, in both directions, we found that the sound velocity decreases with increasing $s_{B}$. However, the sound velocity along the direction $\Gamma J$ decreases faster than in the direction $X \Gamma$. On the other hand, for the configuration $A B_{6}$ we have a different behavior for the sound velocity. In the direction $\Gamma J$, the sound velocity decreases monotonically with increasing $s_{B}$, while along the direction $X \Gamma$ the sound velocity decreases up to $s_{B}=0.00251$, where the minimum sound velocities $v_{T A} / v_{0}=1.07608$ and $v_{T A} / v_{0}=0.40973$ are observed for $m^{*}=s_{B}$ and $m^{*}=1$, respectively.

For the configuration $S(A B)$, the $s_{B}$ dependence of the sound velocity differs completely from the one found for the phases $A B_{2}$ and $A B_{6}$ as shown in Fig. 8. In the direction $\Gamma X$, the velocity increases monotonically with increasing $s_{B}$, while the opposite behavior is found along the direction $M \Gamma$. We were able to fit the sound velocity of the phases $A B_{2}, A B_{6}$, and $S(A B)$ to the expression

$$
v_{T A} / v_{0}=v_{0}+v_{1} s_{B}+v_{2} s_{B}^{2},
$$

where the coefficients $v_{i}$ are reported in Tables II, III, and IV, respectively.

In Figs. 9(a), 10, and 11 the $s_{B}$ dependence of the optical frequencies $\omega_{\mathrm{op}}$ at the $\Gamma$ point is presented for both cases $m^{*}=s_{B}$ and $m^{*}=1$. The optical frequencies are associated with the out-of-phase vibrations of the particles in the unit cell. In general, the number of optical frequencies $n_{\text {op }}$ depends on the number of particles per unit cell $n_{p}$ and the dimensionality of the system, being $n_{\mathrm{op}}=2 n_{p}-2$ for the $2 \mathrm{D}$ colloidal system

TABLE III. Fitting parameters [Eq. (19)] for the sound velocity of the phase $A B_{6}$.

\begin{tabular}{lcccc}
\hline \hline$A B_{6}$ & $m^{*}=s_{B}$ & $m^{*}=s_{B}$ & $m^{*}=1$ & $m^{*}=1$ \\
\hline Direction & $\Gamma J$ & $X \Gamma$ & $\Gamma J$ & $X \Gamma$ \\
$v_{0}$ & 1.289 & 1.288 & 0.485 & 0.476 \\
$v_{1}$ & -176.7 & -142.9 & -65.16 & -50.90 \\
$v_{2}$ & 20861.2 & 24930.7 & 7759.54 & 9060.95 \\
\hline \hline
\end{tabular}


TABLE IV. Fitting parameters [Eq. (19)] for the sound velocity of the phase $S(A B)$.

\begin{tabular}{lcccc}
\hline \hline$S(A B)$ & $m^{*}=s_{B}$ & $m^{*}=s_{B}$ & $m^{*}=1$ & $m^{*}=1$ \\
\hline Direction & $\Gamma X$ & $M \Gamma$ & $\Gamma X$ & $M \Gamma$ \\
$v_{0}$ & 0.001 & 2.015 & -0.021 & 1.417 \\
$v_{1}$ & 13.64 & -2.985 & 10.22 & -1.236 \\
$v_{2}$ & -20.02 & -5.899 & -13.25 & -6.354 \\
\hline \hline
\end{tabular}

at hand. As a general behavior, the optical frequencies for $m^{*}=s_{B}$ are larger than those for $m^{*}=1$.

The phase $A B_{2}$ has 4 optical frequencies and the phase $A B_{6}$ has 12 , since these alloys have three and seven particles per unit cell, respectively. For the colloidal alloys $A B_{2}$ and $A B_{6}$, the different optical frequencies are nondegenerate. The jumps of the optical frequencies for the phase $A B_{2}$ in Fig. 9(a) are associated with the change of the positions of the small particles in the unit cell as a function of $s_{B}$, as can be seen in Fig. 9(b). On the other hand, a different behavior is found for the structure $S(A B)$. In this case, the two allowed optical phonon frequencies are degenerate, which is a consequence of the symmetry of the square lattice structure presented by that phase. The vibrations of the particles in the unit cell are equivalent in both directions.

To conclude, notice that the optical frequencies tend to zero when $s_{B}$ approaches zero only in the case $m^{*}=1$. In this limit $\left(s_{B} \rightarrow 0\right)$ the interaction involving the small particles $B$ becomes negligible, allowing the optical modes to be excited with a very low frequency.

\section{MELTING}

Now we turn our discussion to the melting behavior of the system as a function of the dipole-moment ratio $s_{B}$. The melting temperature will be calculated within the harmonic

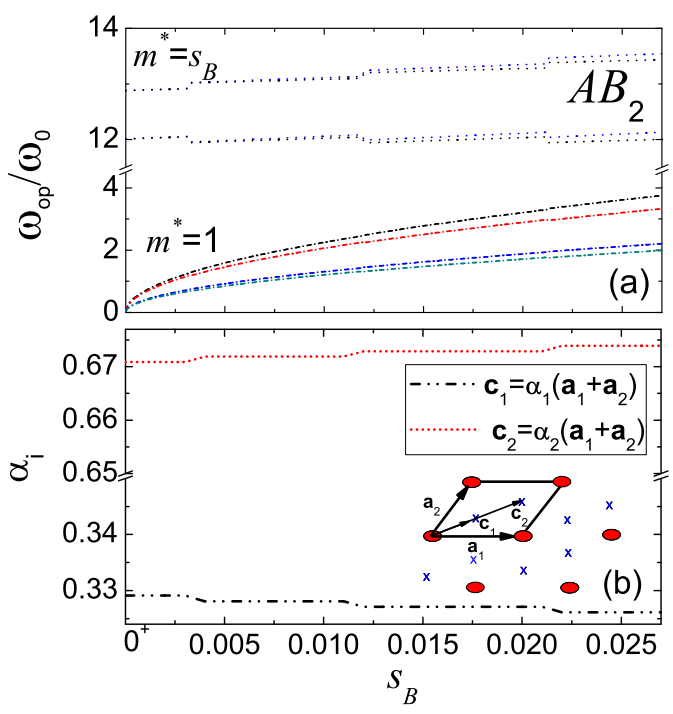

FIG. 9. (Color online) (a) The optical frequencies in units of $\omega_{0}$ at the $\Gamma$ point for $A B_{2}$ as a function of $s_{B}$ for $m^{*}=s_{B}$ (dotted line) and $m^{*}=1$ (short dash dotted line) and (b) positions of the small particles inside the unit cell of the structure $A B_{2}$ as a function of $s_{B}$.
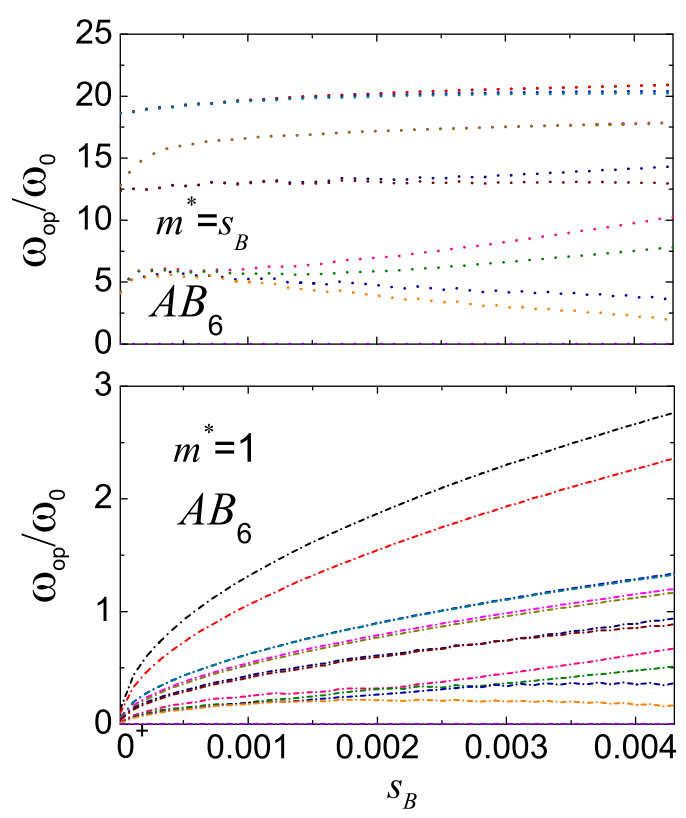

FIG. 10. (Color online) The optical frequencies in units of $\omega_{0}$ at the $\Gamma$ point for $A B_{6}$ as a function of $s_{B}$ for $m^{*}=s_{B}$ and $m^{*}=1$.

approximation using a Lindemann-like criterion. The original Lindemann criterion [26] states that the melting of a given structural phase occurs when the mean-square displacement exceeds a threshold value of the mean interparticle distance $r_{0}[26-28]$ :

$$
\frac{\left\langle u^{2}\right\rangle}{r_{0}^{2}}=\delta^{2},
$$

where the parameter $\delta^{2}$ is obtained numerically from, e.g., molecular dynamics simulation. The symbol \langle\rangle stands for a thermal average. The original Lindemann criterion is not applicable for $2 \mathrm{D}$ crystals because $\left\langle u^{2}\right\rangle$ diverges logarithmically with the size of the system $[19,28]$. Bedanov et al. [28] showed through molecular dynamics simulations that the relative mean-square displacement, given by

$$
\left\langle|\vec{u}(\vec{R})-\vec{u}(\vec{R}+\vec{a})|^{2}\right\rangle
$$

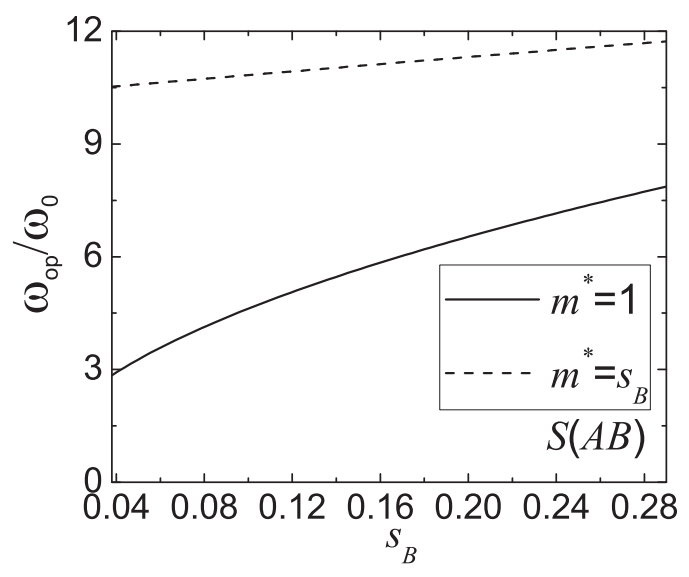

FIG. 11. The optical frequencies in units of $\omega_{0}$ at the $\Gamma$ point for $S(A B)$ as a function of $s_{B}$ for $m^{*}=s_{B}$ and $m^{*}=1$. 
is a well-defined quantity for a 2D infinite system, where $\vec{u}(\vec{R})$ and $\vec{u}(\vec{R}+\vec{a})$ are the displacement vectors at site $\vec{R}$ and at its nearest-neighbor site $\vec{R}+\vec{a}$, respectively, and $\vec{a}$ is the lattice parameter. In Ref. [28], the modified Lindemann-like criterion for $2 \mathrm{D}$ crystals was defined as

$$
\frac{\left\langle|\vec{u}(\vec{R})-\vec{u}(\vec{R}+\vec{a})|^{2}\right\rangle}{r_{0}^{2}}=\delta_{m}^{2},
$$

with the modified Lindemann parameter $\left(\delta_{m}^{2}\right)$ typically $\delta_{m}^{2} \approx$ 0.1 . The melting of the $B$ sub-lattice was studied in Ref. [16] using Monte Carlo simulations and the radial distribution function $g_{B B}(r)$ between the small particles was obtained. For instance, the calculated melting temperature of the $B$ sublattice for the $A B_{2}$ configuration was $4.0 \pm 0.5 \times 10^{-3}$ for $s_{B}=0.025$.

Here we will study the melting behavior of the large $A$ particles. In this case, $r_{0}$ in Eq. (22) is the mean interparticle distance between large particles which is related to the density as $r_{0}=1 / \sqrt{\pi \rho_{A}}$. The parameter $\delta_{m}^{2}$ for 2D dipole interaction [28] is $\delta_{m}^{2}=0.12$, and, therefore, we will take this value in order to determine the melting temperature $\left(T_{M}\right)$ of the $A$ particles. As shown recently, the $B$ sublattice (small particles) is already melted at $T_{M}$, since the melting temperature of the $A$ sublattice (large particles) was estimated to be two orders of magnitude larger than the one for the small particles [16]. In addition, the melting temperature calculated through the harmonic approximation depends on the frequencies of the phonon spectrum which are obtained at $T=0$ by considering both sublattices ordered [e.g., see Eq. (30)]. In the present colloidal alloys the distribution of the small $B$ particles around the big $A$ particles is symmetric. We argue here that since in the melted state the small particles are spread uniformly around the large particles, the effective interaction between small and large particles is very similar to the one found in the crystal structure at $T=0$. In this case, the frequencies of the phonon spectrum obtained at $T=0$ for the ordered arrangement of the colloidal alloy would also in some sense reflect the effective interaction between both types of particles at $T \neq 0$. Therefore, in spite of the $B$ sublattice be already melted at the melting temperature of the $A$ sublattice, we consider the phonon frequencies obtained for the complete ordered structure at $T=0$. We stress that the melting temperature of the large $A$ particles obtained here is only an estimate but we expect that the qualitative trends and the order of magnitude to be correct.

The correlation function $\left\langle|\vec{u}(\vec{R})-\vec{u}(\vec{R}+\vec{a})|^{2}\right\rangle$ is obtained within the harmonic approximation and by considering only the nearest neighbors. In general, each lattice site in the 2D colloidal alloys has several types of nearest neighbors, and the number and the distance of the nearest neighbors depend on the considered colloidal alloy. The melting behavior of the $A$ sublattice will be studied as a function of the dipole-moment ratio $s_{B}$ for the case $m^{*}=1$, i.e., when both types of particles have the same mass [17,29] and for $m^{*}=s_{B}$.

The correlation function between $A$ particles is given by $[19,20]$

$$
\Delta u_{A A}=\frac{1}{N_{A}} \sum_{\alpha=x, y} \sum_{l=1}^{N_{A}}\left\langle\left|u_{\alpha}^{A}(0)-u_{\alpha}^{A}(l)\right|^{2}\right\rangle,
$$

where $u_{\alpha}^{A}(l)$ is the $\alpha$ th component of the displacement vector of the $l$ th nearest neighbor of type $A$ and $N_{A}$ is the number of nearest neighbors of type $A$. For the stable configurations $A B_{2}$ and $A B_{6}$ the $A$ particles are ordered in a hexagonal lattice and therefore each of them has six nearest neighbors. On the other hand, for the phase $S(A B)$ the $A$ particles form a square lattice with each particle having four nearest neighbors.

Using the normal coordinates transformation [20,23],

$$
\begin{aligned}
& u_{\alpha}^{A}(0)=\frac{1}{\sqrt{N m_{A}}} \sum_{\vec{q}, j} e_{\alpha}^{A}(\vec{q}, j) Q(\vec{q}, j), \\
& u_{\alpha}^{A}(l)=\frac{1}{\sqrt{N m_{A}}} \sum_{\vec{q}, j} e_{\alpha}^{A}(\vec{q}, j) Q(\vec{q}, j) e^{i \vec{q} \cdot \vec{R}_{A}(l)},
\end{aligned}
$$

where $m_{A}$ is the mass of the large particle, $N$ the number of unit cells of the crystal, $e_{\alpha}^{A}(\vec{q}, j)$ the $\alpha$ th component of the eigenvector of the $j$ th normal mode of the large particle for the wave vector $\vec{q}, Q(\vec{q}, j)$ the normal coordinate of the vibrational mode, and $\vec{R}_{A}(l)$ the relative vector connecting one $A$ particle at the origin to its $l$ th nearest neighbor of type $A$. From the fact that the thermal average of $Q(\vec{q}, j) Q^{*}\left(\vec{q}^{\prime}, j^{\prime}\right)$ is given by $[20,23]$

$$
\left\langle Q(\vec{q}, j) Q^{*}\left(\vec{q}^{\prime}, j^{\prime}\right)\right\rangle=\frac{k_{B} T}{\omega^{2}(\vec{q}, j)} \delta_{\vec{q} \vec{q}^{\prime}} \delta_{j j^{\prime}},
$$

where $k_{B}$ is the Boltzmann constant and $T$ is the temperature of the system, we obtain

$$
\left\langle\left|u_{\alpha}^{A}(0)-u_{\alpha}^{A}(l)\right|^{2}\right\rangle=\frac{4 k_{B} T}{N m_{A}} \sum_{\vec{q}, j} \frac{\left[e_{\alpha}^{A}(\vec{q}, j)\right]^{2}}{\omega^{2}(\vec{q}, j)} \sin ^{2} \frac{\vec{q} \cdot \vec{R}_{A}(l)}{2} .
$$

Therefore, the expression for $\Delta u_{A A}$ results in

$$
\Delta u_{A A}=\frac{4 k_{B} T}{N m_{A} N_{A}} \Gamma_{A A},
$$

with

$$
\Gamma_{A A}=\sum_{\vec{q}, j} \frac{\left[e_{x}^{A}(\vec{q}, j)\right]^{2}+\left[e_{y}^{A}(\vec{q}, j)\right]^{2}}{\omega^{2}(\vec{q}, j)} \sum_{l=1}^{N_{A}} \sin ^{2} \frac{\vec{q} \cdot \vec{R}_{A}(l)}{2} .
$$

Now the correlation function becomes

$$
\left\langle|\vec{u}(\vec{R})-\vec{u}(\vec{R}+\vec{a})|^{2}\right\rangle=\Delta u_{A A},
$$

and substituting this into the modified Lindemann criterion, we found

$$
\begin{aligned}
\Gamma_{M}= & \frac{4 \pi}{N N_{A} \delta_{m}^{2} \rho_{A}^{3 / 2} a^{3}} \sum_{\vec{q}, j} \frac{\left[e_{x}^{A}(\vec{q}, j)\right]^{2}+\left[e_{y}^{A}(\vec{q}, j)\right]^{2}}{\omega^{2}(\vec{q}, j) / \omega_{0}^{2}} \\
& \times \sum_{l=1}^{N_{A}} \sin ^{2} \frac{\vec{q} \cdot \vec{R}_{A}(l)}{2},
\end{aligned}
$$

where $\omega_{0}^{2}=\mu_{A}^{2} \rho_{A}^{5 / 2} / m_{A}$.

The melting temperature of dipolar systems is usually studied in terms of the dimensionless coupling parameter $\Gamma_{M}=\mu_{A}^{2} / k_{B} T_{M} a^{3}$, which involves the potential and thermal energy. Here we will plot $1 / \Gamma_{M}$ as a function of the dipolemoment ratio $s_{B}$. 


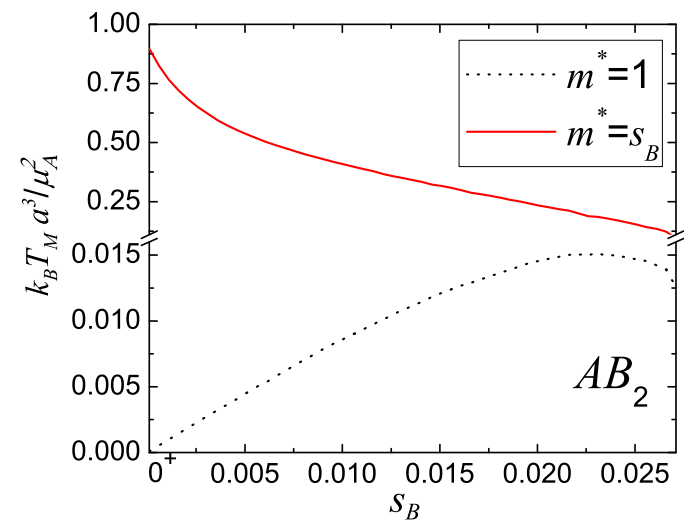

FIG. 12. (Color online) Melting temperature of the $A$ sublattice of the phase $A B_{2}$ as a function of the dipole-moment ratio. $m^{*}=1$ $\left(m^{*}=s_{B}\right)$ means particles $A$ and $B$ with equal (different) masses. For $m^{*}=1$, the melting temperature assumes the maximum value $1 / \Gamma_{M}=0.01507$ for $s_{B}=0.0231$.

In Fig. 12 we present the melting behavior of the structure $A B_{2}(\xi=2 / 3)$ as a function of the dipole-moment ratio $s_{B}$ for the cases with equal $\left(m^{*}=1\right)$ and different $\left(m^{*}=s_{B}\right)$ masses. As commented before, the symbol $0^{+}$(in the $x$ axis of Fig. 12) means that we are not considering $s_{B}=0$ but only $s_{B}$ values very close to zero. Initially, we will focus on the case with $m^{*}=1$. For $s_{B}=0$, i.e., a one-component dipolar system, we found $1 / \Gamma_{M} \approx 0.15$, which is very close to the value $1 / \Gamma_{M} \approx 0.11$ found in Refs. [16] and [30]. Besides, as an important finding, there is an optimum value of the dipole-moment ratio $s_{B}$ for which the melting temperature of the $A$ sublattice reaches a maximum, i.e., for $s_{B}=0.0231$ the melting temperature has the maximum value $1 / \Gamma_{M}=$ 0.01507. This is interesting since it can be used in future experimental studies of 2D binary colloidal systems of dipoles when one wants to maximize the melting temperature. For $s_{B}=0.025$, which is experimentally relevant $[15,16]$, we find that $1 / \Gamma_{M}=1.45 \times 10^{-2}$. It means that, for $s_{B}=0.025$, the

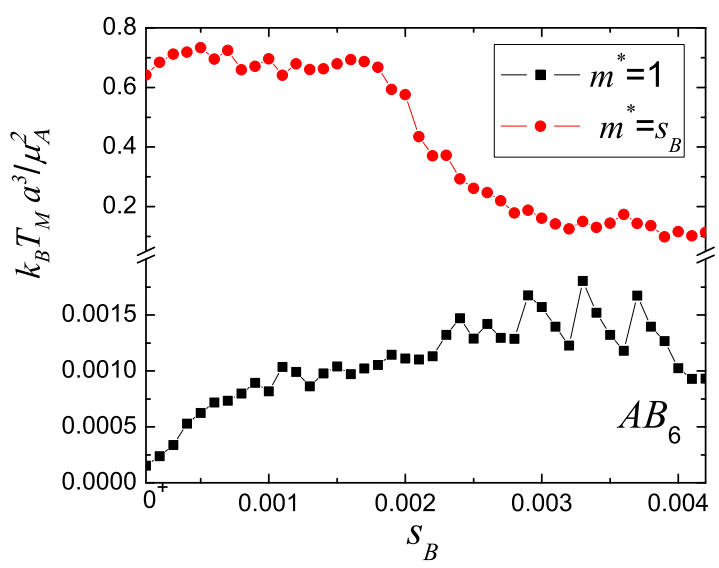

FIG. 13. (Color online) Melting temperature of the $A$ sublattice for the structure $A B_{6}$ as a function of the dipole-moment ratio. $m^{*}=1$ $\left(m^{*}=s_{B}\right)$ means particles $A$ and $B$ with equal (different) masses. The melting temperature for $m^{*}=1$ reaches its maximum value when $s_{B}=0.0033$. melting point of the $A$ sublattice is one order of magnitude larger than that of the $B$ sublattice $\left(1 / \Gamma_{M}=4.0 \pm 0.5 \times 10^{-3}\right)$ calculated using Monte Carlo simulations [16].

In the case of particles having different masses $\left(m^{*}=s_{B}\right)$, we observe a very different qualitative behavior of the melting temperature as a function of $s_{B}$. The melting temperature decreases monotonically as $s_{B}$ is increased. Quantitatively, the melting temperature is more than one order of magnitude larger than for the case with equal masses $m^{*}=1$. The presence of the lighter small dipoles makes the crystalline structure more stable against thermal fluctuations, as compared to the case with $m^{*}=1$.

The melting temperature for the phase $A B_{6}(\xi=6 / 7)$ as a function of $s_{B}$ is presented in Fig. 13 for the cases $m^{*}=1$ and $m^{*}=s_{B}$. The same general qualitative behavior found for the phase $A B_{2}$ is also observed for the phase $A B_{6}$, namely the melting temperature for $m^{*}=1$ exhibits a maximum but now for a smaller $s_{B}=0.0033$, while for the case $m^{*}=s_{B}$ we observe that the melting temperature decreases with increasing $s_{B}$. Also, the melting temperature for $m^{*}=s_{B}$ is about two orders of magnitude larger than that for $m^{*}=1$.

Differently from the smooth behavior found for the phase $A B_{2}$, we observe that for the phase $A B_{6}$ there are fluctuations in the melting temperature curve as a function of $s_{B}$ (Fig. 13) due to numerical inaccuracies. The latter is related to the larger number of particles in the unit cell of the phase $A B_{6}$ [Fig. 1(d)], which increases the number of phonon modes considerably. As is apparent from Fig. 10 there are many low-frequency modes which are more difficult to calculate numerically with high accuracy. And those frequency modes are the ones that contribute strongly to the melting temperature.

The melting of the $A$ sublattice for the structure $S(A B)$ as a function of $s_{B}$, for $m^{*}=1$ and $m^{*}=s_{B}$, is presented in Fig. 14. Unlike the configurations $A B_{2}$ and $A B_{6}$, the phase $S(A B)$ has the same qualitative behavior for $m^{*}=1$ and $m^{*}=s_{B}$. On the other hand, quantitatively, the maximum melting temperature for $m^{*}=1\left(1 / \Gamma_{M} \approx 0.060\right)$ is one order of magnitude smaller than that for $m^{*}=s_{B}\left(1 / \Gamma_{M} \approx 0.138\right)$. This is another example of how the composition changes drastically the properties of the system.

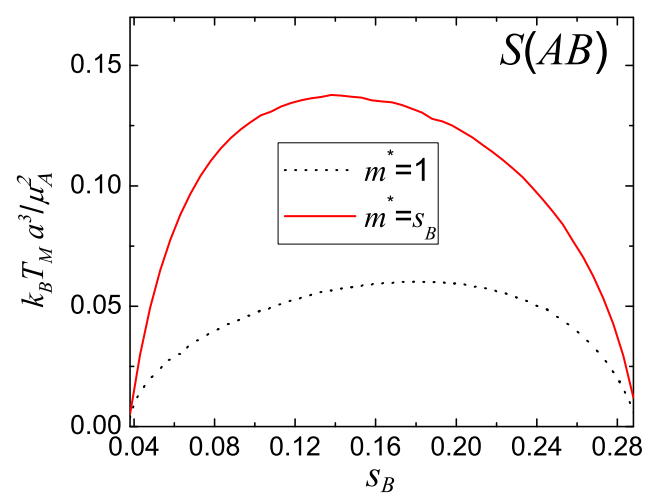

FIG. 14. (Color online) Melting temperature of the $A$ sublattice for the configuration $S(A B)$ as a function of the dipole-moment ratio. Here, for $m^{*}=1$, the maximum temperature $1 / \Gamma_{M} \approx 0.060$ takes place for $s_{B}=0.18$, while for $m^{*}=s_{B}$, the maximum temperature $1 / \Gamma_{M} \approx 0.138$ occurs for $s_{B}=0.138$. 


\section{v. CONCLUSIONS}

We investigated the dynamical properties and melting transition of a 2D binary colloidal system of dipoles interacting through a dipole-dipole repulsive potential. Within the harmonic approximation we calculated the phonon spectra of the system as a function of the relative concentration of small particles, dipole-moment ratio, and mass ratio. We determined the interval of values of the dipole-moment ratio $s_{B}$ for which the colloidal alloys with perfect hexagonal arrangement are stable and have long-range order. For instance, we found that the $A B_{2}$ configuration has long-range order for $s_{B} \lesssim 0.0269$.

For the phase $A B_{5}$ we found that the $T=0$ phonon spectrum consists of imaginary frequencies, indicating that the perfect hexagonal $A B_{5}$ structure is unstable. We did not find a long-range $A B_{6}$ ordered configuration for $s_{B}=0.037$ which is consistent with the experiments reported in Ref. [15].

The gaps in the phonon spectra were analyzed by changing the composition, mass ratio, and the dipole-moment ratio. For example, the phonon gaps of the configurations $A B_{2}$ and $A B_{6}$ are considerably large when the particles have different masses. Furthermore, unlike the colloidal alloys $A B_{2}$ and $A B_{6}$ that have phonon gaps when the particles have different masses as well as equal masses, the configuration $S(A B)$ exhibits phonon gaps only when the particles have different masses. This is an example of how the composition changes the properties of the system. The optical frequencies in the long-wavelength limit were discussed. The number of optical frequencies is associated with the number of particles per unit cell, i.e., the composition. The optical frequencies of the phase $S(A B)$ are degenerate while the ones of the configurations $A B_{2}$ and $A B_{6}$ are not. The common behavior that the optical frequencies go to zero when the dipole-moment ratio tends to zero does not hold when particles have different masses $\left(m^{*}=s_{B}\right)$. We also analyzed the sound velocity of the transverse acoustical mode. As a general behavior, the sound velocity becomes large when the particles have different masses. Furthermore, the speed of sound depends strongly on the composition and the dipole-moment ratio. For instance, for the composition $\xi=6 / 7\left(A B_{6}\right)$ the sound velocity along the $\Gamma X$ direction diminishes only until $s_{B}=0.00251$, where the minimum speed of sound is obtained.

We estimated the melting temperature of the $A$ sublattice as a function of the dipole-moment ratio and composition, within the harmonic approximation, and using the modified Lindemann criterion. For each stable configuration, we determined the value of the dipole-moment ratio for which the melting temperature is a maximum. This is also an important result that will be useful in future experiments of 2D binary colloidal systems of dipoles.

\section{ACKNOWLEDGMENTS}

This work was supported by the Brazilian agencies CNPq (Program Science Without Border), CAPES, and FUNCAP (International cooperation program); the Flemish Science Foundation (FWO-Vl); the bilateral program between Flanders and Brazil (CNPq-FWO collaborating project); and the VLIR-UOS (University Development Cooperation). I.R.O.R. is grateful to Professor E. B. Barros for fruitful discussions. W.P.F. thanks Professor D. Martin A. Buzza for his illuminating comments on this manuscript.
[1] U. Gasser, C. Eisenmann, G. Maret, and P. Keim, Chem. Phys. Chem. 11, 963 (2010).

[2] K. Zahn, R. Lenke, and G. Maret, Phys. Rev. Lett. 82, 2721 (1999).

[3] F. Ebert, P. Dillmann, G. Maret, and P. Keim, Rev. Sci. Instrum. 80, 083902 (2009).

[4] P. Schall, I. Cohen, D. A. Weitz, and F. Spaepen, Nature (London) 440, 319 (2006).

[5] A. M. Alsayed, M. F. Islam, J. Zhang, P. J. Collings, and A. G. Yodh, Science 309, 1207 (2005).

[6] P. Keim, G. Maret, U. Herz, and H. H. von Grünberg, Phys. Rev. Lett. 92, 215504 (2004).

[7] Sofi Nöjd, Priti S. Mohanty, Payam Baheri, Anand Yethiraj, and Peter Schurtenberger, Soft Matter 9, 9199 (2013).

[8] T. Stirner and J. Sun, Langmuir 21, 6636 (2005).

[9] L. Assoud, R. Messina, and H. Löwen, Europhys. Lett. 80, 48001 (2007).

[10] Julia Fornleitner, Federica Lo Verso, Gerhard Kahl, and Christos N. Likos, Soft Matter 4, 480 (2008).

[11] J. Fornleitner, F. Lo Verso, G. Kahl, and C. N. Likos, Langmuir 25, 7836 (2009).

[12] N. Hoffmann, C. Likos, and H. Löwen, J. Phys.: Condens. Matter 18, 10193 (2006).
[13] N. Hoffmann, F. Ebert, C. N. Likos, H. Löwen, and G. Maret, Phys. Rev. Lett. 97, 078301 (2006).

[14] F. Ebert, P. Keim, and G. Maret, Eur. Phys. J. E 26, 161 (2008).

[15] A. D. Law, D. M. A. Buzza, and T. S. Horozov, Phys. Rev. Lett. 106, 128302 (2011).

[16] A. D. Law, T. S. Horozov, and D. M. A. Buzza, Soft Matter 7, 8923 (2011).

[17] J. Fornleitner, G. Kahl, and C. N. Likos, Phys. Rev. E 81, 060401(R) (2010).

[18] L. Bonsall and A. A. Maradudin, Phys. Rev. B 15, 1959 (1977).

[19] G. Goldoni and F. M. Peeters, Phys. Rev. B 53, 4591 (1996).

[20] Xin Lu, Chang-Qin Wu, Andrea Micheli, and Guido Pupillo, Phys. Rev. B 78, 024108 (2008).

[21] I. R. O. Ramos, W. P. Ferreira, F. F. Munarin, G. A. Farias, and F. M. Peeters, Phys. Rev. E 85, 051404 (2012).

[22] G. P. Srivastava, The Physics of Phonons (Adam Hilger, Bristol, 1990).

[23] A. A. Maradudin, E. W. Montroll, G. H. Weiss, and I. P. Ipatova, Theory of Lattice Dynamics in the Harmonic Approximation (Academic Press, New York, 1971), Suppl. 3.

[24] H. H. von Grünberg and J. Baumgartl, Phys. Rev. E 75, 051406 (2007). 
[25] N. Osterman, D. Babic, I. Poberaj, J. Dobnikar, and P. Ziherl, Phys. Rev. Lett. 99, 248301 (2007).

[26] F. A. Lindemann, Physik. Z. 11, 609 (1910).

[27] D. Pines, Elementary Excitations in Solids (W. A. Benjamin, New York, 1963).
[28] V. M. Bedanov, G. V. Gadiyak, and Y. E. Lozovik, Phys. Lett. A 109, 289 (1985).

[29] W. P. Ferreira, G. A. Farias, and F. M. Peeters, J. Phys.: Condens. Matter 22, 285103 (2010).

[30] K. Zahn and G. Maret, Phys. Rev. Lett. 85, 3656 (2000). 